\title{
Zinc deficiency and febrile seizure: a systematic review and meta-analysis
}

\author{
Farhad Heydarian ${ }^{1 \oplus}$, Alireza Ataei Nakhaei ${ }^{1 \oplus}$, Hasan Mehrad Majd ${ }^{20}$, \\ Elham Bakhtiari ${ }^{3 \oplus}$ \\ ${ }^{1}$ Department of Pediatrics, ${ }^{2}$ Clinical Research Unit and ${ }^{3}$ Eye Research Center, Faculty of Medicine, Mashhad University of Medical \\ Sciences, Mashhad, Iran.
}

\begin{abstract}
Background and objectives. Zinc has been reported to be low in children with febrile seizure compared to febrile cases without seizures, but results are inconsistent. A meta-analysis was performed to systematically evaluate the serum level of zinc in febrile children aged between 6-72 months with or without seizures.

Material and methods. A systematic search of databases was performed from January 2000 to January 2019. Studies comparing the serum level of zinc in febrile children with or without seizure were selected.

Results. The major outcome was serum level of zinc. Random effect model was used to calculate pooled standardized mean differences (SMD) with 95\% confidence intervals (CIs). A total of 31 articles were included. Meta-analysis suggested that the serum level of zinc is lower in patients with febrile seizure versus febrile cases without seizure (SMD: $-1.2,95 \% \mathrm{CI}=(-1.47,-0.93)$. In subgroup and sensitivity analysis no significant change was observed in pooled SMD. In meta-regression analysis sample size as a continuous variable had a significant influence on between-study variance $(p=0.02)$. According to cumulative analysis the difference of serum level of zinc in febrile children with or without seizure decreased with time.

Conclusion. This meta-result indicated a significant association of zinc deficiency with seizure in febrile children. It is suspected that decreased level of zinc may be involved in seizure occurrences and it may play a role in the pathogenesis of febrile seizure.
\end{abstract}

Key words: febrile seizure, children, zinc levels.

Seizure is one of the most common lifethreatening events in childhood. ${ }^{1}$ Febrile seizure (FS) has been defined as seizure in correlation with a febrile disease without the infection of the central nervous system (CNS) or acute imbalance of electrolytes in absence of previous afebrile seizures in children aged more than 1 month. The prevalence of FS is 3-5\% worldwide. ${ }^{2,3}$ FS could be simple or complex which is differed in frequency and duration. ${ }^{4}$ Seizure is a result of abnormal and extreme discharge of cortical neurons in the CNS. Neuronal synapses are

$凶$ Elham Bakhtiari

bakhtiarie@mums.ac.ir

Received 30th April 2019, revised 16th July 2019, accepted 18th July 2019. responsible for the composition of excitatory or inhibitory signals in the post-synaptic space as well as chipping in the basal making of neuronal networks in the brain. ${ }^{5}$ Trace elements have several influences in the CNS. They are involved in Ion channels, synaptogenesis, membrane lipid peroxidation, etc. Over the past years, it was considered that trace elements may play a role in the pathogenesis of febrile seizure. It has been suggested that zinc as an essential trace element plays a role in the pathogenesis of seizures. ${ }^{6}$ There are many studies comparing the serum level of zinc in febrile seizure. In some studies levels of zinc in febrile seizure have been reported to be lower when compared with febrile cases without seizures ${ }^{7-9}$ but in some studies no significant difference was reported..$^{10-12}$ 
To the best of our knowledge there is only one systematic review and one meta-analysis evaluating the serum or CSF level of zinc in patients with FS.,13 They reviewed published papers until 2013 and had different inclusion criteria compared with the present study. In one systematic review ${ }^{2}$ the control group included were febrile cases, afebrile seizure and healthy children. On the other hand, there are more than 15 published papers comparing the serum level of zinc in febrile children with or without seizures between the years 2013 and 2018 with different results. ${ }^{7,8,14-22}$ Therefore, the present updated review was carried out to systematically evaluate relevant papers comparing the serum level of zinc in febrile patients with or without seizures until January 2019.

\section{Material and Methods}

\section{Research strategy and study selection}

Published papers were searched by 2 independent researchers (EB and $\mathrm{FH}$ ) in relevant databases including Pubmed, Scopus, Cochrane library and web of science from January 1th, 2000 up to January 31th, 2019. Also e-publications ahead of print were searched in Pubmed. Disagreement between the two independent researchers was verified by the third researcher (AA). The reference lists of all verified studies were also checked to detect additional relevant articles. Searched key words were ["febrile seizure" OR "febrile convulsion"] AND ["level" OR "concentration" OR "profile"] AND ["zinc"] AND ["serum" OR "plasma"] and ["deficiency"]. The present systematic review was conducted according to the standard protocol for meta-analyses of observational studies. Inclusion criteria were original case-control, nested case-control and cross-sectional papers studying febrile children aged 6-72 month with or without seizures without any neurological disorders. Full-text of English articles and English abstract of nonEnglish articles were included. Exclusion criteria were animal or laboratory studies, review studies, randomized clinical trials (RCT), noncontrolled studies, healthy controls or control groups with epilepsy, afebrile seizure or any neurological complications or having received any pharmacological treatment. Articles which did not provide necessary data including patient's age, number of patients in each group and mean \pm SD of zinc level were excluded.

The following information was extracted from each article included: first author, year of publication, country of study, number of subjects in case and control groups, mean $\pm \mathrm{SD}$ of zinc level in both groups, significant difference between groups and method of zinc measurement. Studies were scored according to NOS (Newcastle-Ottawa Quality Assessment Scale Case-Control Studies). ${ }^{23}$ Articles with at least 5 stars were considered as good quality and included in the meta-analysis. The present research was reviewed and approved by Mashhad University of Medical Sciences review board (code: 970646, ethic code: IR.MUMS. MEDICAL.REC.1398). Because the present study was a systematic review and did not relate to patients directly, it was not necessary to provide inform consent form.

\section{Quantitative data synthesis and data analysis}

The data were extracted and meta-analysis was used to pool them. Results were expressed as standardized mean differences (SMD) with 95\% confidence intervals $(95 \% \mathrm{CI})$. If a study only reported the median, range and/or inter-quartile range (IQR); mean and standard deviation (SD) were estimated, as described by Wan et al. ${ }^{24}$ Heterogeneity was checked using $\mathrm{I}^{2}$ statistics. Heterogeneity was considered significant if $\mathrm{I}^{2}$ was more than $50 \%$. In significant heterogeneity, data were analyzed using random effect modelling. ${ }^{25}$ Sensitivity analysis was applied to the evaluation of the results constancy. In order to discover the probable sources of heterogeneity, subgroup analysis, based on study sample size, method of zinc measurement and match of demographic characteristics was performed. Meta-regression was also performed to further explore which variables contribute to 
substantial heterogeneity. Cumulative analysis was also performed to detect the time-based changes in the magnitude of research findings.

Publication bias was also verified by visually check of Begg's funnel plot symmetry and Egger's regression test. ${ }^{26}$ There is significant publication bias if $\mathrm{P}<0.05$. The data were analyzed using comprehensive Meta-analysis software version 2.2.064 (Biostat, Englewood,NJ, USA).

\section{Results}

\section{Search and study selection}

The primary literature search retrieved 558 articles. Some articles were removed because they were not original studies; some were excluded because of duplication. Some articles were excluded, because they were books, book section, review paper or animal studies. Some studies were excluded because they had no control group or they had a control group with different inclusion criteria. Finally, 31 articles were included in the present review..$^{7-12,14-22,}$ 27-42 All were case-control or cross sectional. The diagram of the study selection process is presented in Figure 1. Details of studies included are presented in Table I.

\section{Serum level of zinc}

Serum level of zinc was reported in 31 studies. Totally 3642 febrile children with or without seizures were included as FS and febrile group without seizure. In the FS group there were 1803 patients and in the febrile group without seizure there were 1839 patients. A significant lower serum level of zinc in febrile seizure versus febrile children without seizure was reported in 27 studies. ${ }^{7-9,14-22,28-42}$ In 4 studies no significant difference between groups was reported. ${ }^{10-12,27}$ Totally 31 studies were included in the meta-analysis. ${ }^{7-12,14-22,27-42}$ Meta-analysis using random effect modelling, suggested that patients with FS had a significantly lower serum level of zinc in comparison with febrile patients without seizure (SMD: -1.2, 95\% CI:
(-1.47, -0.93), P value<0.001, $\left.\mathrm{I}^{2}: 92.62 \%\right)$. Forest plot is shown in Figure 2.

\section{Heterogeneity analysis}

To assess the probable sources of heterogeneity in the results, subgroup, meta-regression and sensitivity analysis were carry out. In the subgroup analysis, the SMD of serum levels of zinc did not differ substantially according to sample size, method of zinc measurement or matching of demographic characteristics (Table II). Higher serum levels of zinc were observed in the febrile seizure patients compared with febrile cases without seizure across all subgroups. To additionally explore the potential sources of heterogeneity within the results; meta-regression analysis was performed by considering all the potential factors including sample size, method of zinc measurement and matching of demographic characteristics. According to the metaregression analysis, sample size $(p=0.02)$ was found to be a significant contributing factor for

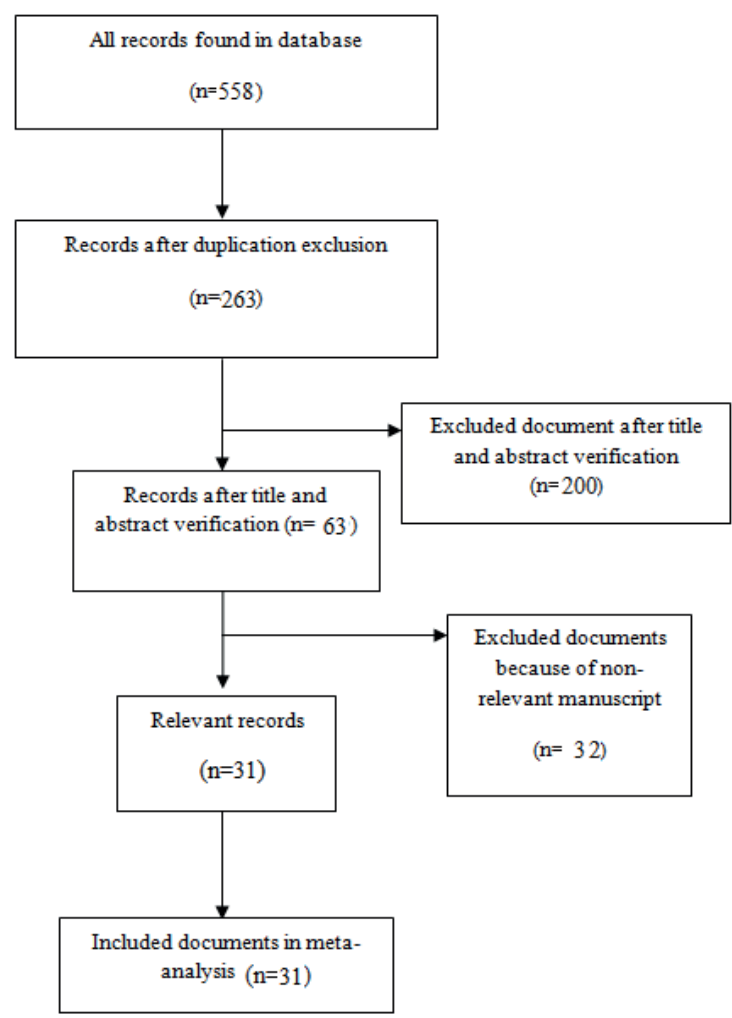

Fig. 1. Diagram of study selection. 


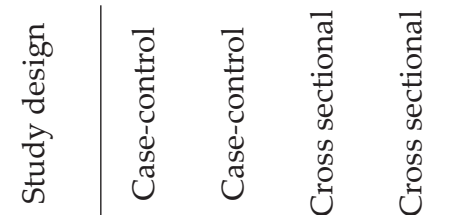
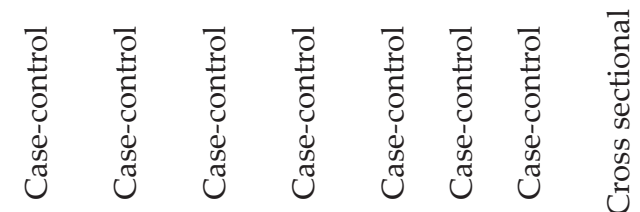

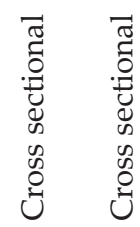

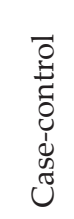

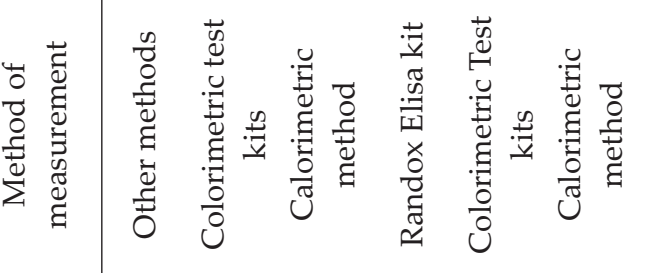

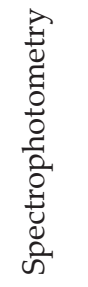

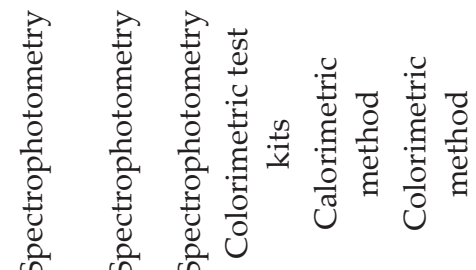

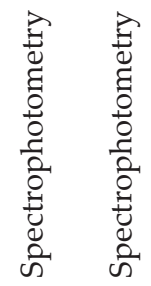

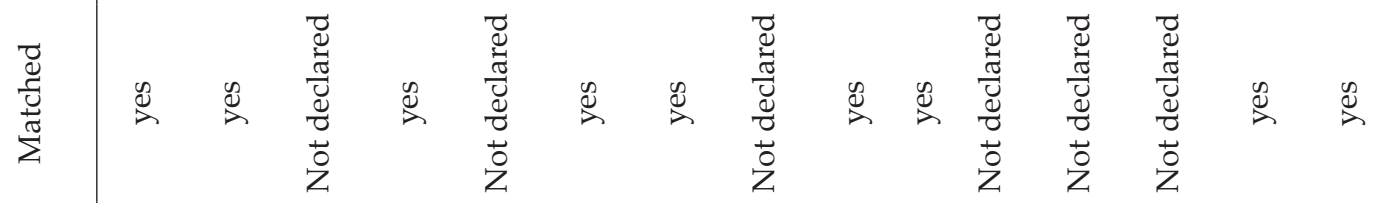

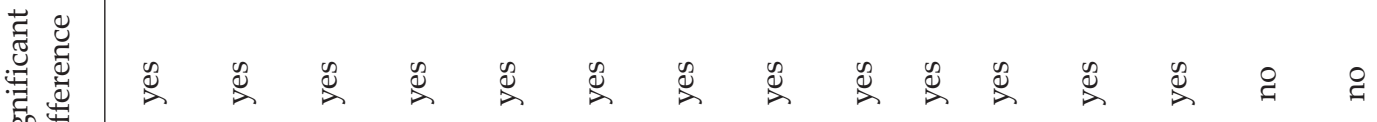
जे

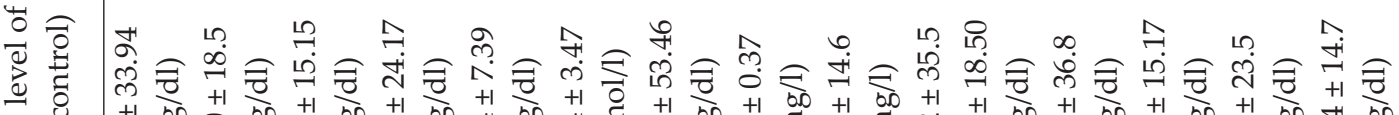

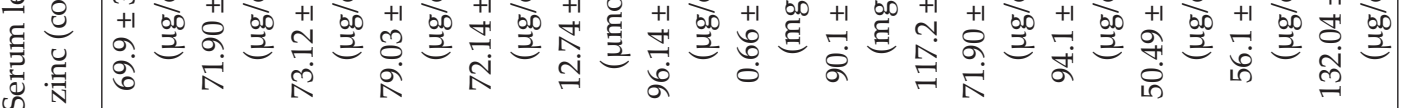

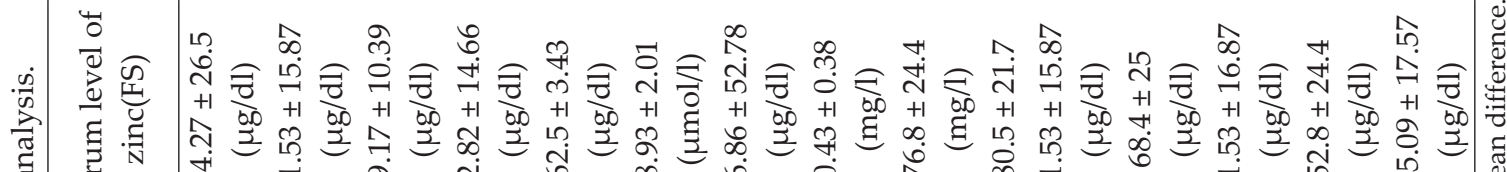
จ कृ

है जै

ิำ $\infty$ ๙

近亲

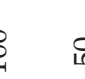



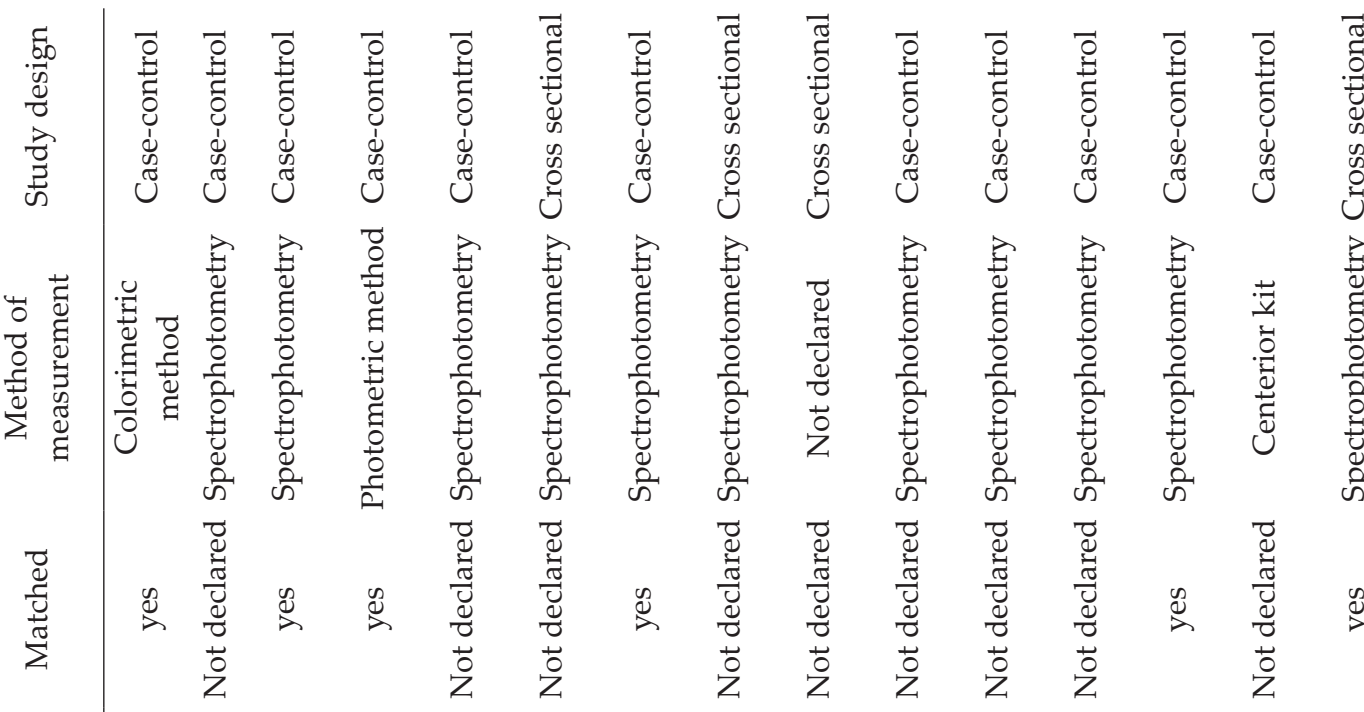

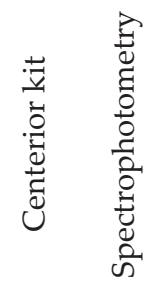

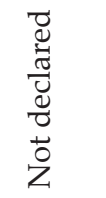

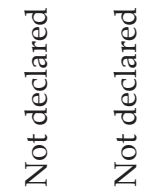

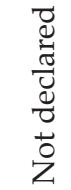

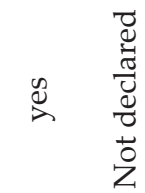

节芯

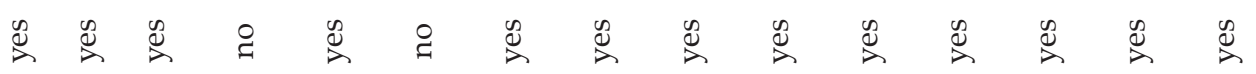

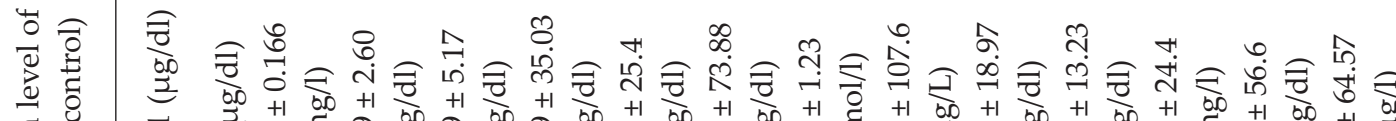

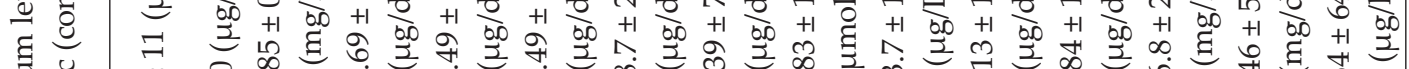

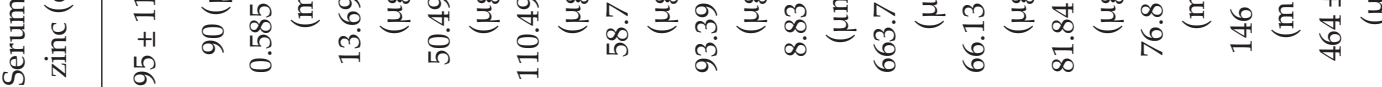

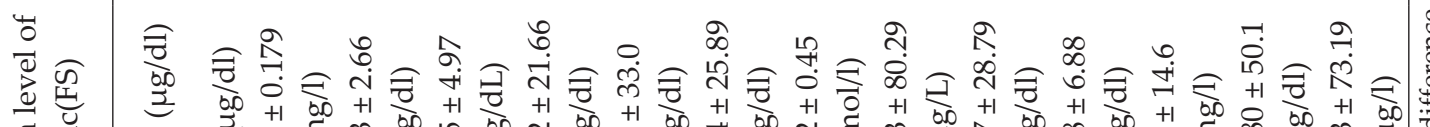

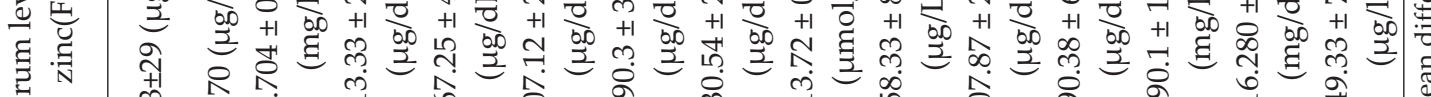
矛 $\begin{aligned} & \text { ती } \\ & \text { ก }\end{aligned}$

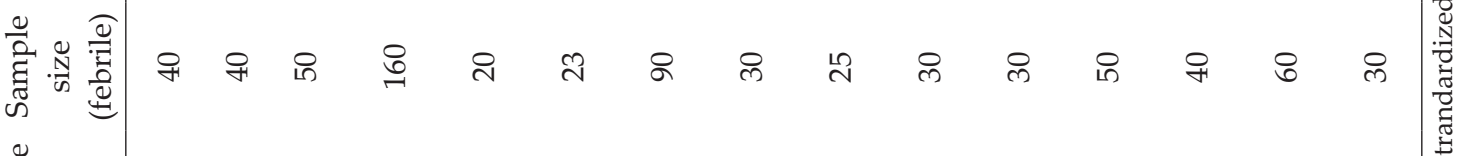

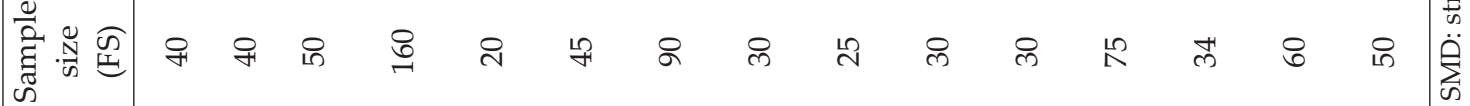

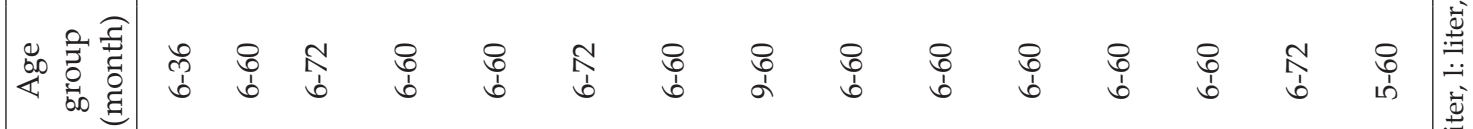

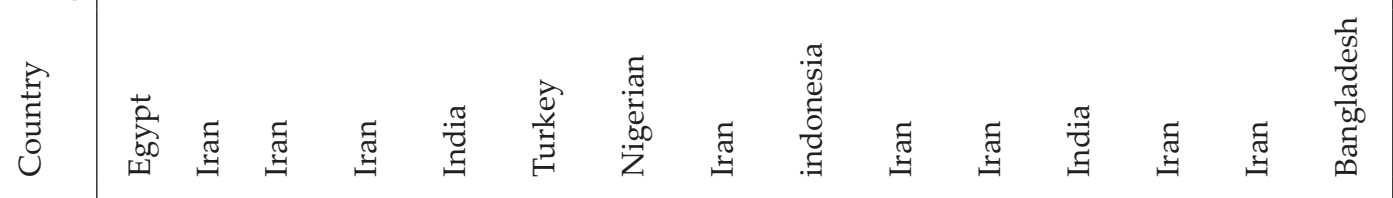

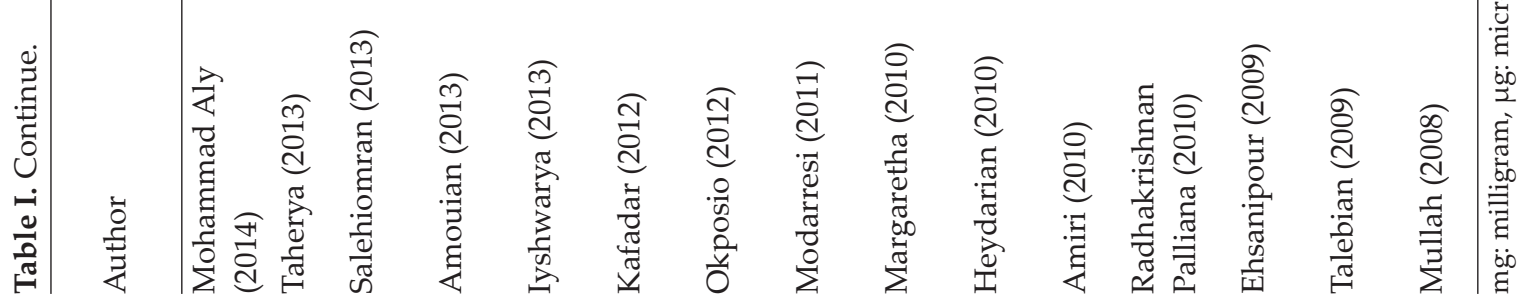


Studies

El-masry 2018

Hubaira 2018

Sampathkumar 2018

Maheshwari 2018

Pravin Soni 2017

Nemichandra 2017

khajeh 2016

Shokrzadeh 2016

Bonu 2016

Namakin 2016

Gatto 2015

Pannerselvam 2015

Sreenivasa 2015

N. Salah 2014

Joshi 2014

Mohammad Aly 2014

Salehiomran 2013

Amoulan 2013

Iyshwarya 2013

Taherya 2013

Kafadar 2012

Okposio 2012

Modarresi 2011

Margaretha 2010

Heydarian 2010

Amiri 2010

Radhakrishnan Palliana 2010

Ehsanipour 2009

Talebian 2009

Mullah 2008

Ganesh 2008
Estimate (958 C.I.)

$-0.834(-1.291,-0.377)$

$-0.599(-0.883,-0.316\rangle$

$-1.012(-1.265,-0.758\rangle$

$-0.806(-1.178,-0.434\rangle$

$-1.668(-1.962,-1.374)$

$-1.337(-1.676,-0.999)$

$-0.730\langle-1.252,-0.207\rangle$

$-0.611(-0.906,-0.316)$

$-0.648(-1.087,-0.209)$

$-1.237(-1.674,-0.801)$

$-0.599(-0.883,-0.316\rangle$

$-0.811(-1.218,-0.403\rangle$

$-0.683(-1.086,-0.280)$

$-0.135\langle-0.756,0.485\rangle$

$-1.437(-1.877,-0.997)$

$-1.897(-2.424,-1.369\rangle$

$-0.684(-1.087,-0.281)$

$0.137(-0.083,0.356)$

$-3.239(-4.182,-2.297)$

$-2.311(-2.877,-1.745)$

$0.107(-0.396,0.609)$

$-1.069(-1.381,-0.756)$

$-0.662(-1.182,-0.143\rangle$

$-5.197(-6.357,-4.037)$

$-0.984(-1.520,-0.448)$

$-1.690(-2.279,-1.100)$

$-0.762(-1.132,-0.392)$

$-0.668(-1.138,-0.198)$

$-0.553(-0.917,-0.188)$

$-4.166(-4.954,-3.377)$

$-3.351\langle-4.048,-2.654\rangle$

Overall $\left(1^{\wedge} 2=92.62 \%, \mathrm{P}<0.001\right)-1.204 \quad(-1.471,-0.937)$

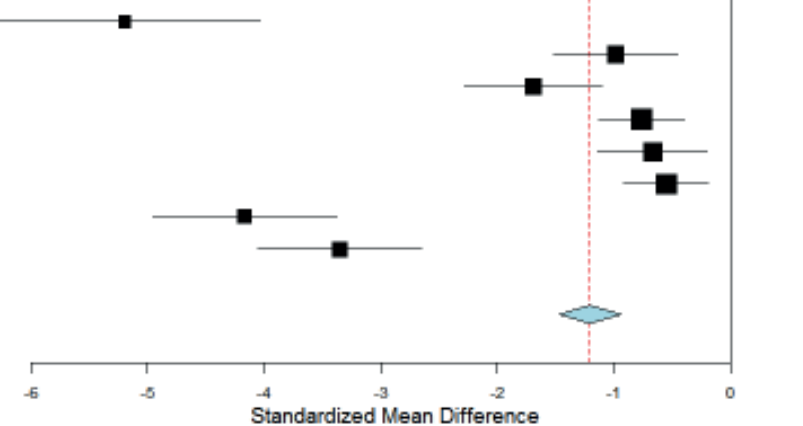

Fig. 2. The serum level of zinc in febrile children with or without seizure in pooled SMD.

Table II. Subgroup analysis of articles included according to sample size, method of zinc measurement and matched demographic characteristics.

\begin{tabular}{|c|c|c|c|c|c|c|}
\hline \multirow{2}{*}{ Sub groups } & \multirow{2}{*}{$\begin{array}{l}\text { No. of } \\
\text { studies }\end{array}$} & \multicolumn{2}{|c|}{$\begin{array}{l}\text { Difference between FS and } \\
\text { febrile group without seizure }\end{array}$} & \multicolumn{3}{|c|}{ Test of heterogeneity } \\
\hline & & SMD (95\% CI) & $\mathrm{p}$ value & Model & $\mathrm{I}^{2}(\%)$ & $\mathrm{P}$ value \\
\hline \multicolumn{7}{|l|}{ Sample size } \\
\hline Less than 100 & 16 & $-1.66(-2.21,-1.1)$ & $<0.001$ & $\mathrm{R}$ & 93.67 & $<0.001$ \\
\hline 100 or more & 15 & $-0.82(-1.08,-0.57)$ & $<0.001$ & $\mathrm{R}$ & 89.53 & $<0.001$ \\
\hline \multicolumn{7}{|c|}{ Method of zinc measurement } \\
\hline Spectrophotometry & 18 & $-1.29(-1.67,-0.9)$ & $<0.001$ & $\mathrm{R}$ & 91.58 & $<0.001$ \\
\hline Other methods & 13 & $-1.08(-1.46,-0.7)$ & $<0.001$ & $\mathrm{R}$ & 93.08 & $<0.001$ \\
\hline \multicolumn{7}{|c|}{ Demographic chracteristics matching } \\
\hline Matched & 16 & $-1.17(-1.57,-0.76)$ & $<0.001$ & $\mathrm{R}$ & 93.75 & $<0.001$ \\
\hline Not declared & 15 & $-1.23(-1.59,-0.87)$ & $<0.001$ & $\mathrm{R}$ & 91.38 & $<0.001$ \\
\hline
\end{tabular}

FS: febrile seizure, SMD: strandardized mean difference, CI: confidence interval, R: random. 
between study variance for SMD analysis but not method of zinc measurement $(p=0.82)$ and matching of demographic characteristics $(p=$ 0.58).

In the sensitivity analysis the effect of each study on the overall pooled SMD was assessed. According to sensitivity analysis no significant change in the direction of pooled SMD was observed, indicating the stability and robustness of the results. Forest plot is shown in Figure 3.

\section{Cumulative analysis}

In cumulative analysis studies were added according to date of publication and the results were summarized as each new study was added.
According to cumulative analysis the difference of serum level of zinc in febrile children with or without seizures decreased over time. Forest plot is shown in Figure 4.

\section{Publication bias}

Begg's funnel plot and Egger's regression test are usually used to determine publication bias of the included studies. Egger's test revealed significant asymmetry ( $p$ value $=0.0006$ ). Funnel plot is shown in Figure 5.

\section{Discussion}

Presently, clinical evidence support the relationship between zinc deficiency and

Studies
Overall
- El-masry
- Hubaira
- Sampathkumar
- Maheshwari
- Pravin Soni
- Nemichandra
- khajeh
- Shokrzadeh
- Bonu
- Namakin
- Gatto
- Pannerselvam
- Sreenivasa
- N. Salah
- Joshi
- Mohammad Aly
- Salehiomran
- Amouian
- lyshwarya
- Taherya
- Kafadar
- Okposio
- Modarresi
- Margaretha
- Heydarian
- Amiri
- Radhakrishnan Pallana
- Ehsanipour
- Talebian
- Mullah
- Ganesh

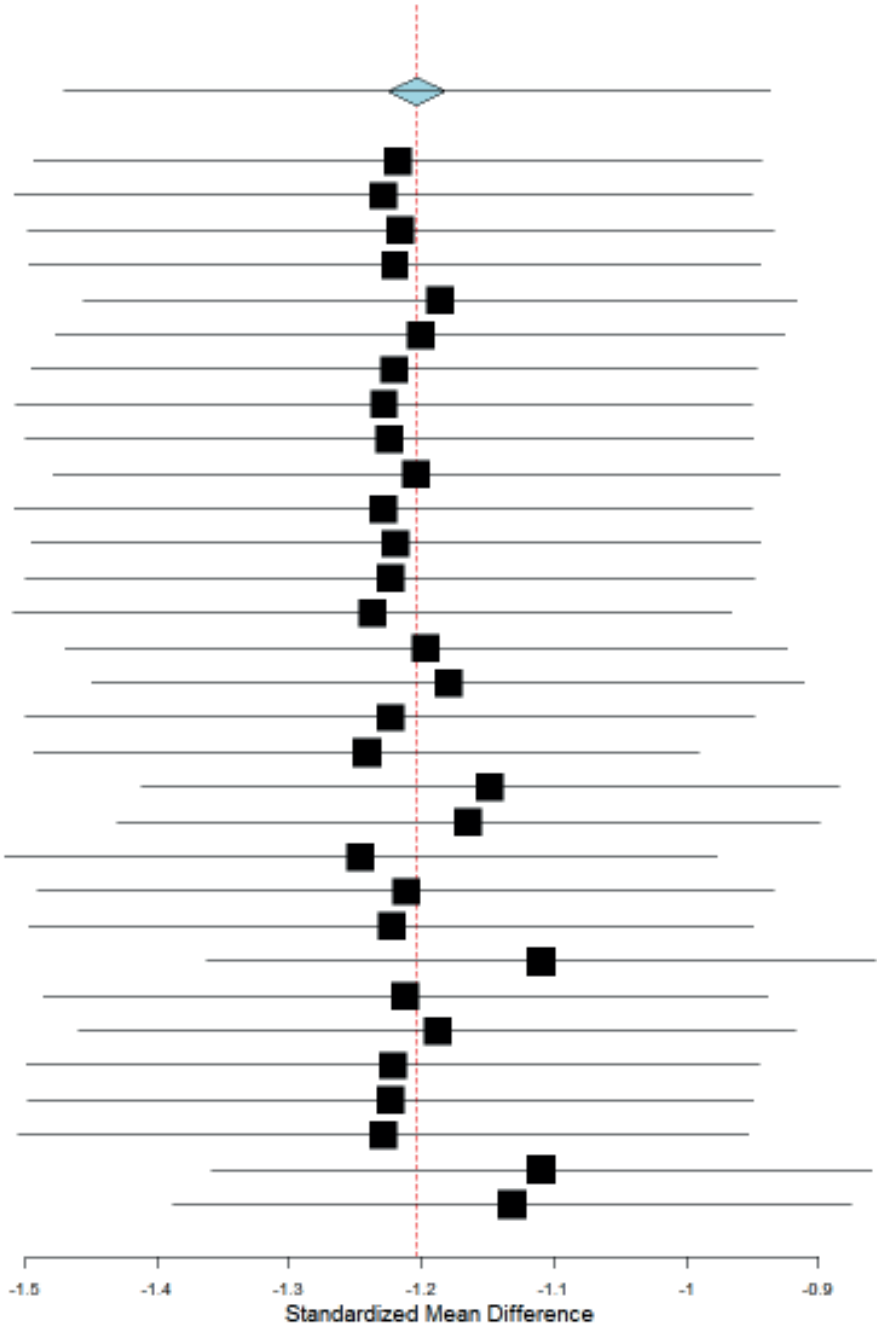

Fig. 3. Sensitivity analysis of included studies. 


\section{Mullah}

+ Ganesh

+ Ehsanipour

+ Talebian

+ Margaretha

+ Heydarian

+ Amiri

+ Radhakrishnan Palliana

+ Modarresi

+ Kafadar

+ Okposio

+ Salehiomran

+ Amouian

+ lyshwarya

+ Taherya

+ N. Salah

+ Joshi

+ Mohammad Aly

+ Gatto

+ Pannerselvam

+ Sreenivasa

+ khajeh

+ Shokrzadeh

+ Bonu

+ Namakin

+ Pravin Soni

+ Nemichandra

+ El-masry

+ Hubaira

+ Sampathkumar

+ Maheshwari
$-4.166(-4.954,-3.377)$

$-3.737(-4.534,-2.939)$

$-2.712(-4.971,-0.453)$

$-2.154(-3.766,-0.543)$

$-2.733(-4.362,-1.105)$

$-2.423(-3.724,-1.121)$

$-2.306(-3.406,-1.206)$

$-2.089(-3.001,-1.178)$

$-1.919(-2.730,-1.108)$

$-1.712(-2.480,-0.943)$

$-1.632(-2.283,-0.982)$

$-1.541(-2.127,-0.954)$

$-1.407(-1.983,-0.831)$

$-1.530(-2.108,-0.952)$

$-1.586(-2.155,-1.016)$

$-1.493(-2.037,-0.950)$

$-1.487(-2.002,-0.972)$

$-1.509(-2.009,-1.010)$

$-1.450(-1.906,-0.994)$

$-1.412(-1.843,-0.982)$

$-1.372(-1.780,-0.964)$

$-1.340(-1.731,-0.948)$

$-1.299(-1.666,-0.933)$

$-1.268(-1.619,-0.918)$

$-1.265(-1.603,-0.926)$

$-1.281(-1.613,-0.950)$

$-1.281(-1.599,-0.963)$

$-1.262(-1.570,-0.955)$

$-1.234(-1.527,-0.941)$

$-1.220(-1.497,-0.944)$

$-1.204(-1.471,-0.937)$

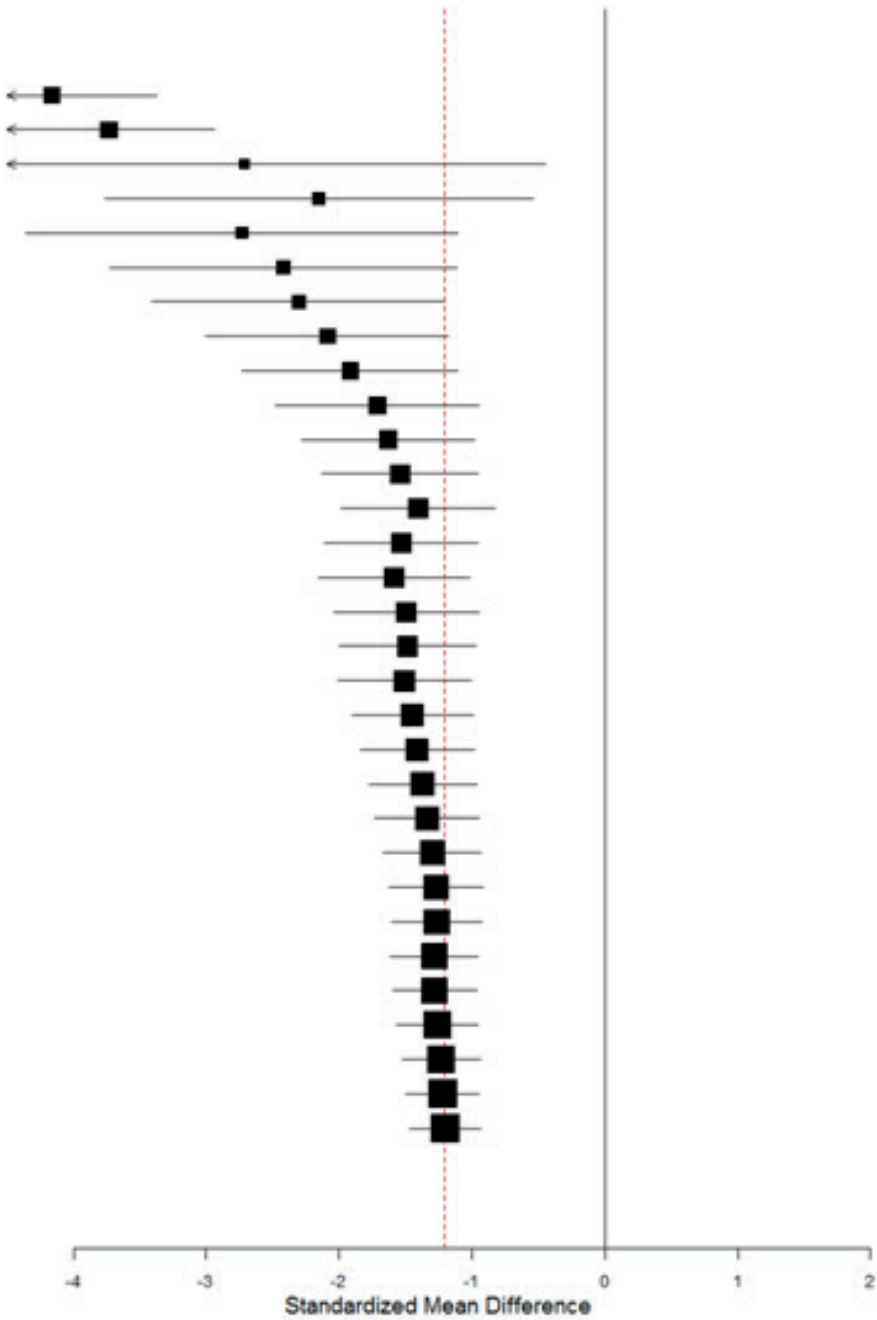

Fig. 4. Cumulative analysis of included studies.

seizure occurrence in febrile children. Therefore clinical data comparing the serum level of zinc in febrile children with or without seizures were systematically reviewed in the present study. According to a meta-analysis the serum level of zinc was significantly lower in FS children compared with febrile cases without seizure. In subgroup and sensitivity analysis no significant change was observed in pooled SMD. Sample size as continuous variable had a significant influence on between-study variance in the meta-regression analysis.

Daily intake of zinc is necessary because there is no storage system for zinc. Sources of zinc include whole grains, cheese, meat, shellfish and legumes. ${ }^{43}$ Many people in developing countries suffer from zinc deficiency, including approximately $79 \%$ in south Asia. ${ }^{44}$

Zinc is one of the essential trace elements in the body, an especially high level is required in the human brain. It is involved in many biological activities, metabolism and differentiation of cells. It is also effective in normal functioning and development of brain cells. ${ }^{45}$ Zinc has also been recognized as a cofactor in the synthesis and secretion of neurotransmitters. ${ }^{46}$ Pyridoxal phosphate (PP) is an essential cofactor for the biosynthesis of neurotransmitters in brain neurons. Hippocampus with nearly $30 \mu \mathrm{g} / \mathrm{g}$ dry weight has the most amount of zinc in brain tissues. Zinc is essential for the synthesis of PP. ${ }^{47}$ Imbalance of zinc leads to 


\section{Funnel Plot of Precision by Std diff in means}

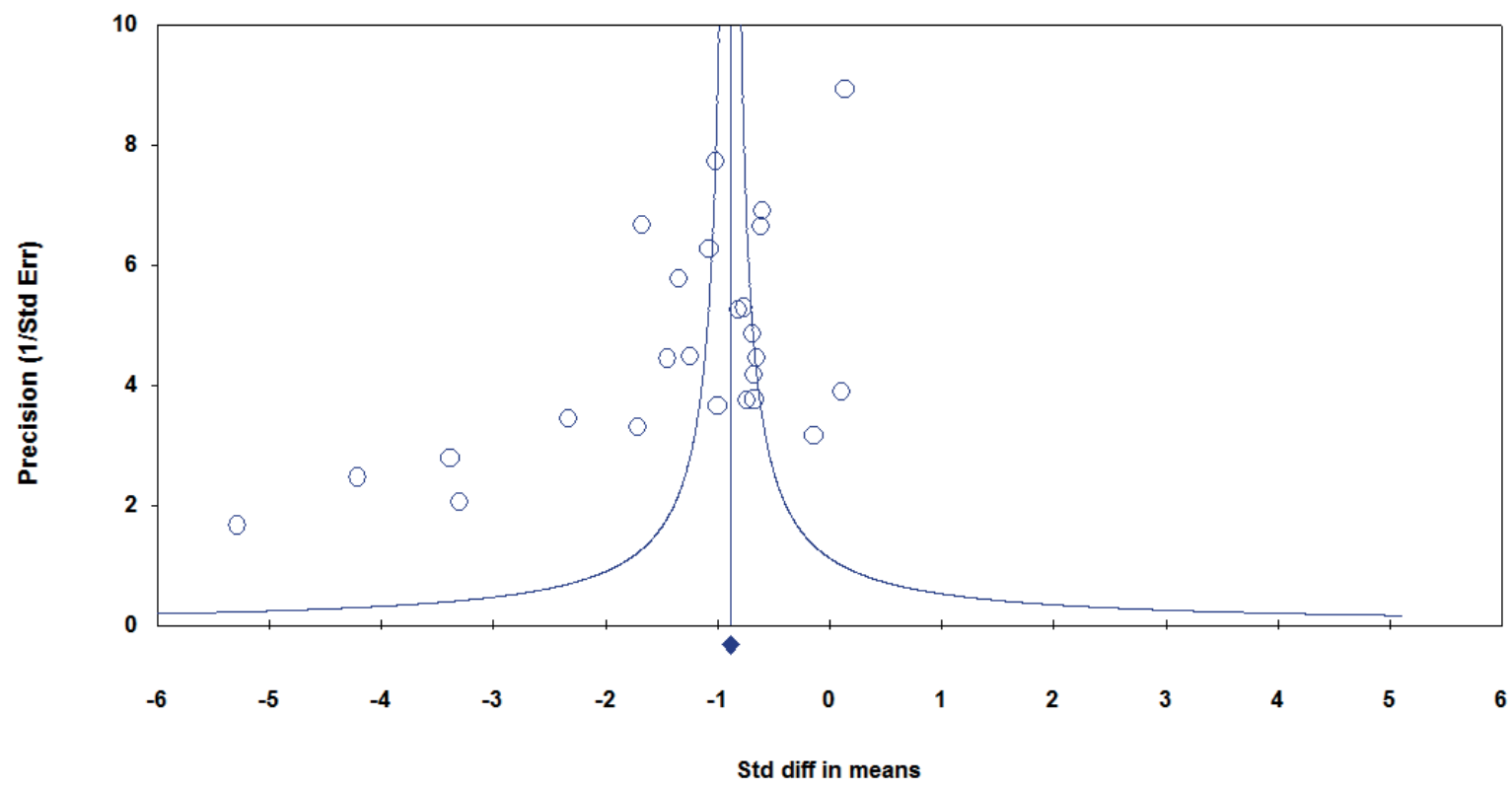

Fig. 5. The funnel plot of publication bias in pooled SMD analysis.

neurodegenerative disorders, oxidative stress, etc. Also the function of some proteins involved in seizures is affected by zinc imbalance. Some excitatory mechanisms can be suppressed by zinc. Zinc is a regulator of a rate-limiting enzyme, glutamic acid decarboxylase in the synthesis of gamma aminobutyric acid (GABA). Decrease of zinc leads to N-Mothy1-D-Asparate (NMDA) receptors activation, GABA inhibition and changes in calcium channel function. These changes could lead to seizure occurrence. ${ }^{48-50}$ GABA brain level is reduced due to low serum level of zinc and as a result, threshold of seizure occurrence is reduced. Serum level of zinc can be affected by fever and acute phase response during infectious diseases. It is suggested that cytokines such as interleukin 6, interferon, tumor necrosis factor and interleukin 1 can reduce serum levels of zinc during infections. ${ }^{51}$ So in cases with borderline serum level of zinc, infections may trigger hypozincemia.

As reviewed in the present study, the relationship between zinc and seizures was studied. To date, only one systematic review and one meta-analysis evaluating the serum or CSF levels of zinc in febrile seizures has been published, but the inclusion criteria were different. ${ }^{2,13}$ Additionally, more than 15 papers have been published between 2013 and 2018 with different results. Therefore, we felt a new systematic review and meta-analysis was necessary.

Between studies heterogeneity was a common finding in the meta-analyses. In the present study, the meta-analysis showed significant heterogeneity in the pooled SMD analysis. Sensitivity analysis, subgroup analysis and meta-regression were used to discover the possible causes of between-study heterogeneity as well as heterogeneity decrement. The results of the present review were robust. According to sensitivity analysis any single study did not affect the estimated pooled SMD. Metaregression confirmed that the sample size of the study had a significant effect on the pooled SMD and could be considered as a confounder but not the method of zinc measurement and group matching. Cumulative analysis showed a decreasing trend in the difference of serum levels of zinc in febrile children with or without seizures during the time. Despite dietary intake and supplementation reducing 
micronutrient and nutrient deficiencies, remain a common problem in developing countries. Therefore, preventative zinc supplementation may outweigh any potentially adverse effects in areas where risk of zinc deficiency is high.

This meta-result indicated a significant association of zinc deficiency with seizures in febrile children. It is suspected that decreased level of zinc may be involved in seizure occurrence and it may play a role in the pathogenesis of febrile seizure.

The major limitations of the present study were: A) In spite of an attempt for a comprehensive search, it may be the case that some articles were missed. B) Despite subgroup analysis, significant heterogeneity was seen and could have influenced the results. C) Potential confounders were not verified in almost all of the studies so we were unable to analyze them. D) Publication bias may have affected the pooled results. E) Most included studies were case-controlled. It is well known that casecontrolled studies have unavoidable limitations such as selection bias in case and control groups and many other confounders which may affect the pooled results.

\section{Acknowledgments}

This work was supported by Research Affairs Office of Mashhad University of Medical Sciences, Mashhad, Iran (Grant number: 970646).

\section{REFERENCES}

1. Sadleir LG, Scheffer IE. Febrile seizures. BMJ 2007; 334: 307-311.

2. Nasehi MM, Sakhaei R, Moosazadeh M, Aliramzany M. Comparison of serum zinc levels among children with simple febrile seizure and control group: a systematic review. Iran J Child Neurol 2015; 9: 17-24.

3. Heydarian F, Vatankhah H. The role of anemia in first simple febrile seizure in children aged 6 months to 5 years old. Neurosciences (Riyadh) 2012; 17: 226229.
4. Salzmann A, Guipponi M, Lyons PJ, et al. Carboxypeptidase A6 gene (CPA6) mutations in a recessive familial form of febrile seizures and temporal lobe epilepsy and in sporadic temporal lobe epilepsy. Human Mutat 2012; 33: 124-135.

5. Rolls ET, Treves A. Neural Networks and Brain Function. Oxford: Oxford University Press, 1998.

6. Mathie A, Sutton GL, Clarke CE, Veale EL. Zinc and copper: pharmacological probes and endogenous modulators of neuronal excitability. Pharmacol Ther 2006; 111: 567-583.

7. Taherya M, Kajbaf TZ, Janahmadi N, Malamiri RA, Musavi MB. Serum zinc level in children with simple febrile convulsions. Iran Red Cresc Med J 2013; 15: 626-627.

8. Hubaira, Wani ZA, Qadri SMR. Relationship between serum zinc levels and simple febrile seizures: hospital based case control study. Int J Contemp Pediatr 2018; 5: 42-45.

9. Maheshwari N, Omperkash, Shaikh M, Hingorjo B, Kazi Y, Rehman A. Febrile seizures; serum zinc level in children with reporting at a tertiary care hospital of Sindh. Proffesional Med J 2018; 25: 461-465.

10. Amouian S, Mohammadian S, Behnampour N, Tizrou M. Trace elements in febrile seizure compared to febrile children admitted to an academic hospital in Iran, 2011. J Clin Diagn Res 2013; 7: 2231-2233.

11. Kafadar I, Akıncı AB, Pekun F, Adal E. The role of serum zinc level in febrile convulsion etiology. J Pediatr Inf 2012; 6: 90-93.

12. Salah ON, Abdelraouf ER, Abdelhameed MH, et al. Assessment of the level of GABA and some trace elements in blood in children who suffer from familial febrile convulsions. OA Maced J Med Sci 2014; 2: 68-73.

13. Saghazadeh A, Mahmoudi M, Meysamie A, Gharedaghi M, Zamponi GW, Rezaei N. Possible role of trace elements in epilepsy and febrile seizures: a meta-analysis. Nut Rev 2015; 73: 760-779.

14. Sampathkumar P, Kannan KS. A comparative study of serum zinc levels in children with febrile seizures and children with fever without seizures in an urban referral hospital. Int J Contemp Pediatr 2018; 5: 977982.

15. Soni SP, Une L. Role of serum zinc level in simple febrile seizures: a hospital based study from rural area of Maharashtra. Med Pulse Int J Pediatr 2017; 3: $36-38$

16. Nemichandra SC, Prajwala HV, Harsha S, Narayanappa D. Implications of alteration of serum trace elements in febrile seizures. Int J Curr Res 2017; 9: 55044-55047. 
17. Khajeh A, Miri-Aliabad G, Fayyazi A, Safdari Z, Keikha M, Askari H. Serum zinc level in children with febrile convulsion. Zahedan J Res Med Sci 2016; 18: e5881.

18. Shokrzadeh M, Abbaskhaniyan A, Rafati M, Mashhadiakabr M, Arab A. Serum zinc and copper levels in children with febrile convulsion. Pharm Biomed Res 2016; 2: 19-24

19. Bonu S, Mohanty AA, Mishra PR. Serum zinc level in children with febrile convulsions and its comparision with that of control group. Yuva J Med Sci 2016; 2: 133-135.

20. Namakin K, Zardast M, Sharifzadeh G, Bidar T, Zargarian S. Serumtrace elements in febrile seizure: a case-control study. Iran J Child Neurol 2016; 10: 57-60.

21. Gattoo I, Harish R, Quyoom Hussain SQ. Correlation of serum zinc level with simple febrile seizures: a hospital based prospective case control study. Inter J Pediatr 2015; 3: 509-515.

22. Sreenivasa B, Kumar S, Manjunatha B. Role of zinc in febrile seizures. Int J Contemp Pediatr 2015; 2: 137-140.

23. Margulis AV, Pladevall M, Riera-Guardia N, et al. Quality assessment of observational studies in a drug-safety systematic review, comparison of two tools: the Newcastle-Ottawa scale and the RTI item bank. Clin Epidemiol 2014; 6: 359-368.

24. Wan X, Wang W, Liu J, Tong T. Estimating the sample mean and standard deviation from the sample size, median, range and/or interquartile range. BMC Med Res Methodol 2014; 14: 135.

25. Borenstein M, Hedges LV, Higgins JP, Rothstein HR. A basic introduction to fixed-effect and randomeffects models for meta-analysis. Res Synth Methods 2010; 1: 97-111.

26. Peters JL, Sutton AJ, Jones DR, Abrams KR, Rushton L. Comparison of two methods to detect publication bias in meta-analysis. JAMA 2006; 295: 676-680.

27. Joshi SS. Assessment of serum iron and zinc status in febrile seizures-A prospective case control study. J Dent Med Sci 2014; 13: 51-89.

28. Salehiomran MR, Mahzari M. Zinc status in febrile seizure: a case-control study. Iran J Child Neurol 2013; 7: 20-23.

29. Iyshwarya U, Pawan Kalyan PS, Suma HR, Prabhavati, Aruna Kumari R. Serum trace elements and oxidative stress marker in children with febrile seizure. J Biomed Sci 2013; 2. doi: 10.3823/1013.
30. Okposio MM, Sadoh WE, Ofovwe GE, Onyiriuka AN. Serum zinc level in Nigerian children with febrile convulsion. J Pediatr Neurol 2012; 10: 187191.

31. Modarresi MR, Mahmoodian T. Yaghini O, Shahabi J, Moasaiiebi D, Shahkarami SMA, The relationship between zinc deficiency and febrile convulsion in Isfahan, Iran. Iran J Child Neurol 2011; 5: 29-33.

32. Margaretha L, Masloman N. Correlation between serum zinc level and simple febrile seizure in children. Paediatr Indones 2010; 50: 326-330.

33. Heydarian F, Ashrafzadeh F, Ghasemian A. Serum zinc level in patients with simple febrile seizure. Iran J Child Neurol 2010; 4: 41-44.

34. Amiri M, Farzin L, Moassesi ME, Sajadi F. Serum trace element levels in febrile convulsion. Biol Trace Elem Res 2010; 135: 38-44.

35. Palliana RR, Singh DK, Ashwin B. Zinc deficiency as a risk factor for febrile seizure. Pediatric Oncall J 2010; 7: 104-105.

36. Ehsanipour F, Talebi-Taher M, Harandi NV, Kani K Serum zinc level in children with febrile convulsion and its comparison with that of control group. Iran J Pediatr 2009; 19: 65-68.

37. Ganesh R, Janakiraman L. Serum zinc levels in children with simple febrile seizure. Clin Pediatr (Phila) 2008; 47: 164-166.

38. Mollah MAH, Rakshit SC, Anwar KS, et al. Zinc concentration in serum and cerebrospinal fluid simultaneously decrease in children with febrile seizure: findings from a prospective study in Bangladesh. Act Pædiatr 2008; 97: 1707-1711.

39. Pannerselvam K, Raju P, Mani S, Reddy SM, Sekar P. Serum zinc levels in children with simple febrile seizures. Int J Contemp Pediatr 2017; 2: 424-427.

40. Talebian A, Vakili Z, Talar SA, Kazemi SM, Mousavi GA. Assessment of the relation between serum zinc \& magnesium levels in children with febrile convulsion. Iran J Pathol 2009; 4: 157-160.

41. Aly IAERM, Kmal HM, Soliman DR, Mohamed MH. Iron profile parameters and serum zinc and copper levels in children with febrile convulsions in Banha. J Am Sci 2014; 10: 320-327.

42. El-Masry HMA, Sadek AA, Hassan MH, Ameen $\mathrm{HH}$, Ahmed HA. Metabolic profile of oxidative stress and trace elements in febrile seizures among children. Metab Brain Dis 2018; 33: 1509-1515.

43. Ma J, Betts NM. Zinc and copper intakes and their major food sources for older adults in the 1994-96 continuing survey of food intakes by individuals (CSFII). J Nutr 2000; 130: 2838-2843. 
44. Akhtar S. Zinc status in South Asian populations-an update. J Health Popul Nutr 2013; 31: 139-149.

45. Bitanihirwe BK, Cunningham MG. Zinc: the brain's dark horse. Synapse 2009; 63: 1029-1049.

46. Offringa M, Newton R.Prophylactic drug management for febrile seizures in children. Cochrane Database Syst Rev 2012: CD003031.

47. Mishra OP, Singhal D, Upadhyay RS, Prasad R, Atri D. Cerebrospinal fluid zinc, magnesium, copper and gamma-aminobutyric acid levels in febrile seizures. J Pediatr Neurol 2007; 5: 39-44.
48. Gower-Winter SD, Levenson CW. Zinc in the central nervous system: from molecules to behavior. Biofactors 2012; 38: 186-193.

49. Plum LM, Rink L, Haase H. The essential toxin: impact of zinc on human health. Inter J Environ Res Public Health 2010; 7: 1342-1365.

50. Takeda A. Zinc homeostasis and functions of zinc in the brain. Biometals 2001; 14: 343-351.

51. Foster M, Samman S. Zinc and regulation of inflammatory cytokines: implications for cardiometabolic disease. Nutrients 2012; 4: 676-694. 